DOI: 10.21608/zvjz.2017.28663.

\title{
Assessment of the Duration of Maternal Antibodies Specific to Live Attenuated peste des petits Ruminant Virus Vaccine in Lambs and Kids
}

\author{
Ayman A-Shehata ${ }^{1}$, Elshaima M. Fawzi ${ }^{1 *}$, Afaf M. Menaze ${ }^{1}$, Mohamed I. Essa ${ }^{1}$, Mervat M. \\ Mahmoud $^{2}$. \\ ${ }^{1}$ Animal Medicine Department, Division of Infectious Diseases, Faculty of Veterinary Medicine, \\ Zagazig University, 44511, Egypt \\ ${ }^{2}$ Virology Department, Animal Health Research Institute, Dokki, Giza, Egypt
}

\begin{abstract}
Maternal immunity in lambs and kids born from vaccinated dams with peste des petits ruminant (PPR) live attenuated cell culture vaccine was evaluated by using competitive ELISA (c-ELISA). Serum samples were collected from each dam prior to the vaccination to detect the titre of antibody against the infection with PPRV and from lambs and kids on the day of birth, then every 15 days until reach 4.5 months of age to determine the level of maternal antibodies against PPR virus transferred from vaccinated dams to their newly born lambs and kids. Results showed that the antibody titer averages in lambs and kids reach the maximum at one month of age (OD average below 20) then, declined gradually at 105 days in which $80 \%$ of kids still have OD average below 50 while $40 \%$ of lambs are still protected. So, we recommended the application of vaccine to newly born lambs and kids from immunized dams at 3 months of age by live attenuated PPR vaccine strain Egypt/87 grown on the Vero cell culture.
\end{abstract}

Keywords: Small ruminants, PPRV- vaccine, Maternal Immunity, c-ELISA.

\section{Introduction}

Peste des petits ruminants (PPR) are highly contagious acute viral disease affecting both domesticated and wild small ruminants [1]. The infection of cattle, pigs and rats is thought to be subclinical [2]. The name PPR (small ruminants plague) was demonstrated by Francophone West Africa and secondly kills a huge number of sheep and goats [3]. The disease is characterized clinically by fever, necrotic stomatitis, anorexia, respiratory distress, occulonasal discharge and diarrhea [4].

PPR is one of fundamental causes hinders the productivity of goats and sheep and associated with high mortality and morbidity rates of affected small ruminants [5]. The disease is caused by peste des petits ruminants virus (PPRV) as an enveloped RNA virus belong to Family Paramyxoviridae, Genus Morbilli viruses, is closely related to measles virus (MV), canine distemper (CDV) virus and rinderpest virus (RPV) [6].

The disease is common in developing countries due to depending on small ruminants rearing and trading for food supply [7]. Since 2007, about one billion in Africa and Asia small ruminants had been thought to be at risk of infection with PPRV [8]. PPR is one of notifiable disease of major economic importance. The PPR characterize by a sudden incidence of illness and associated with restrictions of movements of both animals and its products [9]. Zaher and Ahmed [10] collected Samples from 270 animals (170 Sheep and 100 goats) from Al Daqhaliya,Al Qalyoubiya and Sharkia governorates, Egypt. Rounding and syncytial formation of Vero cells are detected in coincidence with the result of ELISA which revealed that positivity of $56 \%$ of examined goats and $35.29 \%$ of examined sheep against PPRV.

Libeau et al. [11] developed a competitive ELISA as a rapid and sensitive method to detect level of antibodies against infection with PPRV which depends on the recombinant PPRV nucleoprotein. This nucleoproteinis antigenically highly conserved within Morbilli viruses, and is more immunogenic so it was used for the detection of PPRV in spite of its internal location. The competitive ELISA was compared with viral neutralization test (VNT). The VNT is slow and only can differentiate between the cross reactive (RPV) and PPRV while, c-ELISA was more rapid, highly 
sensitivity (94.5\%) and specificity (99.4\%) than VNT, in addition to the its used antigen is safe and can be produced in large amount in vitro.

El-Allawy et al. [12] reported that a total of 658 sheep and 622 goat sera were collected from Assiut, Sohag and Quena Governorates. Samples were screened for neutralizing antibodies to PPR and rinderpest (RP) viruses. Neutralizing antibodies against both viruses were found in sheep and goat sera. All sera were tested for detection of specific antibodies against PPRV only, using ELISA and an agar gel precipitation test but ELISA was found to be the most sensitive one.

Farmers in areas where the virus is endemic are unable to achieve the strict sanitary control measures. Therefore, the control of PPR requires an effective vaccine and for this purpose several vaccines including both homologous and recombinant vaccines have been developed [13].

The age of newly born lambs and kids to be vaccinated depend widely on the amount of maternal antibodies received so the duration of maternal antibodies varies from several days to weeks be studied by many authors to evaluate more or less exact time of start the vaccination [14-18]. Antibodies of maternal origin were detected in young animals until 180 days of age but the threshold level of protection was assed at 135 and 105days in kids and lambs, respectively. Lambs and kids from naturally infected dams or vaccinated one should be revaccinated at 150 and 120days of age, respectively [14]. Sayem [15] and Ahmed et al. [16] demonstrated that Kids of vaccinated dams were advisable to be vaccinated after 4 months of age due to decrease in maternal antibodies. Sanne et al. [17] studied the passive immunity transmitted to 112 new born lambs from pregnant ewes vaccinated with homologous Nigeria 75/1 PPR vaccine at 3 and 4 months of pregnancy was analyzed using c-ELISA. Also, it was observed that level of passive immunity of examined lambs from 15 days of birth and every 15 days up to 150 days was declined about $70 \%$ at day 75 and $95 \%$ at day 90 after birth, so it is recommended to vaccinate lambs against PPR within 2.5 to 3 months of age. The present study was conducted to evaluate the level of antibodies of maternal origin in both kids and lambs born to vaccinated dams and the efficacy of the passive immunity against PPRV by c-ELISA to determine the preferable time to start the vaccination.

\section{Material and Methods}

\section{Animals}

Twelve kids and 10 lambs born from susceptible pregnant five goats and five sheep vaccinated with PPR lives attenuated tissue culture virus vaccine. Good healthy five kids and five lambs were selected for assessment of maternal immunity.

\section{Vaccination of dams}

Serum samples from each dam were collected prior to the vaccination to determine antibody titer against PPRV. There is only one PPR vaccine commercially available in Egypt which is live attenuated lyophilized-Egypt/87 PPR vaccine grown on the Vero cell culture.

At 3 months of pregnancy, dams were vaccinated with $1 \mathrm{ml}$, subcutaneously of homologous PPR lyophilized vaccine. Pregnant vaccinated dams were clinical examined during pregnancy period. Serum samples were taken after 15 days after vaccination of pregnant dams to check the seroconversion. An adequate amount of colostrums was fed to young lambs and kids.

\section{Collection and preparation of samples}

Serum samples were collected from five kids and five lambs born from vaccinated dams (five goats and five sheep) on the zero days (birthday), within intervals of 15 days until 135 days of age $(0,15,30,45,60,75$ days...etc), to determine the maternal antibodies against PPRV transferred from vaccinated dams to their newly born kids and lambs. Serum samples were stored at $-20^{\circ} \mathrm{C}$ until being examined by c-ELISA.

\section{PPRV antibodies in serum samples were carried out by Competitive}

Serum samples from kids and lambs were tested by c-ELISA [ID screen PPR competition ELISA kit (id-vet. CIRADEMVT, Montpellier, France)].

The contents of kit are distilled water (30ml), Microplates coated with PPR 
nucleoprotein, Negative Control, Positive Control, Dilution Buffer 13, Dilution Buffer 4, Wash concentrate (20X), Wash concentrate (20X), Substrate Solution (TMB), Stop Solution and Concentrated Conjugate (10X) for sheep and goats c-ELISA was outlined as the method developed by Libeau et al. [11]. Dilution of the conjugate $10 \mathrm{x}$ to $1 \mathrm{x}$ in dilution buffer $4.9 \mathrm{ml}$ dilution buffer 4 was added to $1 \mathrm{ml}$ conjugate $10 \mathrm{x}$ as each plate needed approximately $10 \mathrm{ml}$ of conjugate $1 \mathrm{x}$.

Washing solution $20 \mathrm{x}$ was diluted to $1 \mathrm{x}$ using distilled water, $10 \mathrm{ml}$ of' washing solution 20x were added to $190 \mathrm{ml}$ of distilled water as each plate needed approximately 200 $\mathrm{ml}$ washing solution $1 \mathrm{x}$. To deactivate nonspecific viral inhibitors, serum samples were inactivated for $30 \mathrm{~min}$ in a water bath by heat at $56^{\circ} \mathrm{C}$. All reagents and serum samples should take the room temperature before use. Twenty-five microliters of dilution buffer 13 were added to each well. Twenty-five microliters of positive control were added to well A1 and B1. Twenty-five microliters of the negative control were added to well $\mathrm{C} 1$ and D1. Twenty-five microliters of each serum sample were added to each of the remaining wells. The plate was incubated at $37^{\circ} \mathrm{C}$ for 45 minutes. Each well was washed 3 times with $300 \mathrm{ul}$ of washing solution 1x. 100 microliters of Conjugate $1 \mathrm{x}$ were added to each well. The plates were incubated at $21^{\circ} \mathrm{C}$ for 30 minutes. Each well was washed 3 times with $300 \mathrm{ul}$ of washing solution $1 \mathrm{x} .100$ microliters of substrate solution were added to each well. The plates were incubated for 15 minutes at $21^{\circ} \mathrm{C}$. 100 microliters of stop solution were added to each well. The optical density was read at wavelength $450 \mathrm{~nm}\left(\mathrm{OD}_{450}\right)$.

The validity of the test was assessed by calculating the mean value of control negative OD (which must be more than 0.7) and dividing the mean value of control positive OD by the mean value of control negative OD which must be less than 0.3 .

In the c-ELISA, the competition percentage (Sample/Negative \% [SN \%]) of each serum sample was calculated according to the following formula:

SN $\%=$ OD sample/OD negative control $\mathrm{x} 100$ 1 - Less than or equal to $50 \%$ are considered positive

2- More than $60 \%$ are considered negative

\section{Results}

No specific clinical signs of any diseases were showed in kids and lambs during the examination period. Kids and lambs were regularly examined during the period of samples collection.

The collected serum samples from 10 vaccinated dams (5 goats, 5 sheep) were tested using c-ELISA technique. High level of antibodies titre of PPRV was obtained, this mean that all dams exposed to the vaccine were sero-converted.

Table 1: Sample/Negative (SN \%) in kids by using c-ELISA.

\begin{tabular}{|c|c|c|c|c|c|c|c|c|c|c|}
\hline \multirow{2}{*}{ Animal number } & \multicolumn{10}{|c|}{ Days after birth } \\
\hline & $\mathbf{0}$ & 15 & 30 & 45 & 60 & 75 & 90 & 105 & 120 & 135 \\
\hline 1 & 110 & 9 & 10 & 11 & 29 & 29 & 32 & 41 & 45 & 78 \\
\hline 2 & 77 & 8 & 7 & 11 & 32 & 38 & 48 & 71 & 89 & - \\
\hline 3 & 87 & 11 & 8 & 12 & 27 & 29 & 35 & 35 & 42 & 80 \\
\hline 4 & 83 & 24 & 8 & 28 & 34 & 36 & 41 & 44 & 86 & - \\
\hline 5 & 95 & 9 & 6 & 19 & 28 & 30 & 37 & 40 & 47 & 88 \\
\hline Average SN \% & 90.4 & 12.2 & 7.8 & 16.2 & 30 & 32.4 & 38.6 & 46.4 & 61.8 & 82 \\
\hline
\end{tabular}

Table 2: Sample/Negative (SN \%) in lambs by using c-ELISA.

\begin{tabular}{|c|c|c|c|c|c|c|c|c|c|}
\hline \multirow{2}{*}{ Animal number } & \multicolumn{9}{|c|}{ Days after birth } \\
\hline & 0 & 15 & 30 & 45 & 60 & 75 & 90 & 105 & 120 \\
\hline 1 & 70 & 10 & 9 & 20 & 39 & 43 & 54 & 88 & - \\
\hline 2 & 95 & 8 & 9 & 27 & 41 & 44 & 53 & 71 & - \\
\hline 3 & 72 & 9 & 9 & 19 & 30 & 39 & 44 & 45 & 98 \\
\hline 4 & 102 & 11 & 8 & 10 & 25 & 33 & 33 & 81 & - \\
\hline 5 & 89 & 8 & 5 & 15 & 20 & 38 & 41 & 44 & 110 \\
\hline
\end{tabular}




\begin{tabular}{llllllllll} 
Average SN\% & 85.6 & 9.2 & 8 & 18.2 & 31 & 39.4 & 45 & 65.8 & 104 \\
\hline
\end{tabular}

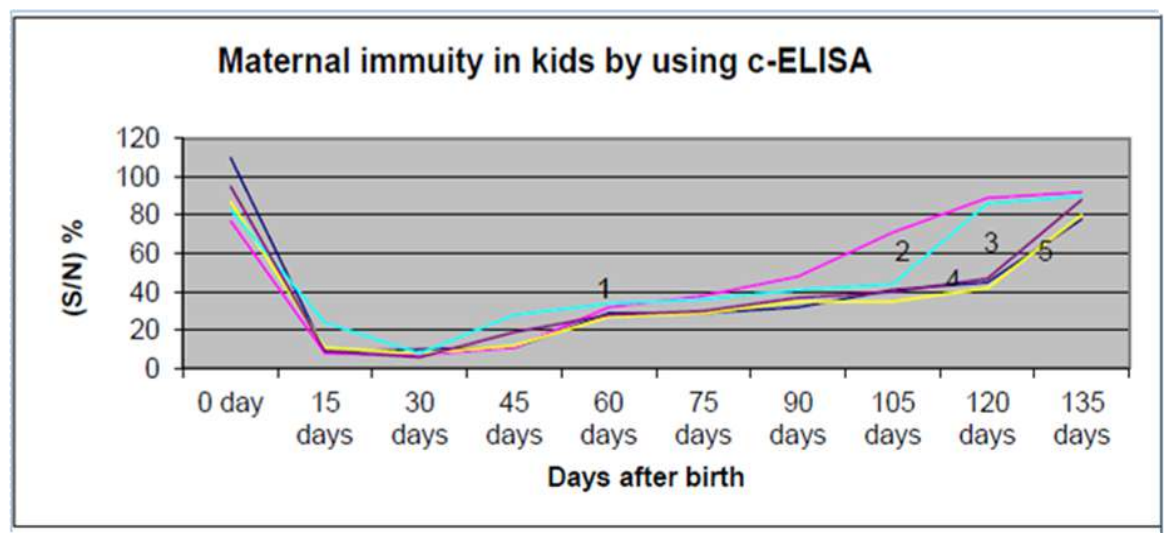

Figure 1: Maternal immunity against PPRV using c-ELISA in kids- Average of Sample/Negative (S/N \%) for the five kids was calculated and represented against days after birth.

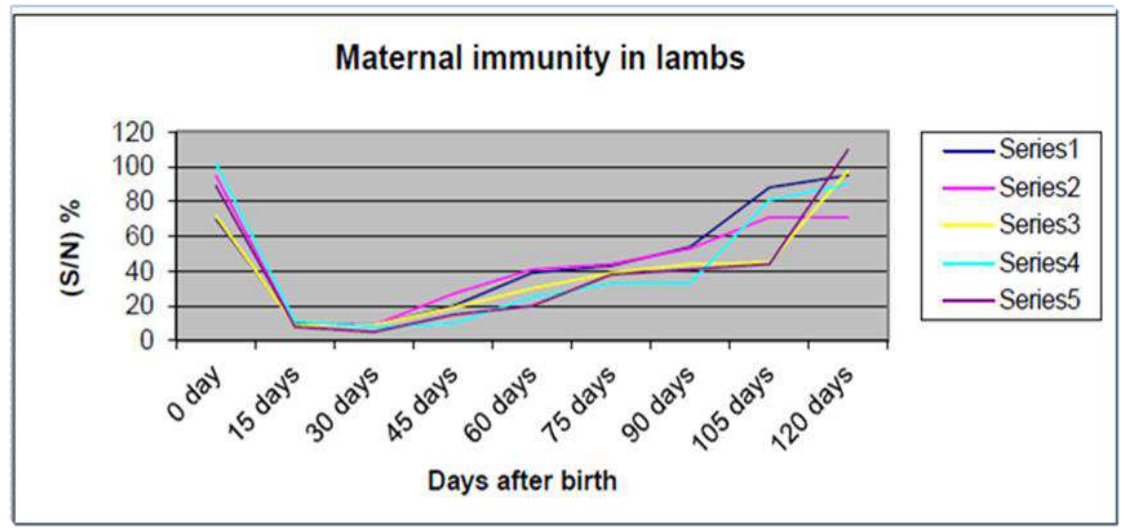

Figure 2: Maternal immunity against PPRV using c-ELISA in lambs- Average of Sample/Negative (S/N \%) for the five lambs was calculated and represented against days after birth.

The collection of serum samples collected from kids and lambs were done from the day zero of birth until 135 days post-birth and tested by c-ELISA showed that the highest titer averages in kids and lambs were at one month of age then, decline gradually were shown in Tables $(1,2)$, alternatively.

At 105 days of age, one kid (out of 5 kids) was negative. At 120 days after vaccination, 2 kids became unprotected. All kids showed negative antibody titer at 4.5 months of age as shown in Figure (1).

Out of 5 lambs, 2 lambs were below the protective level at 3 months. At 3.5 months of age 3 lambs became unprotected and were below the protective level (0.5) according to [11], all lambs were negative at 4 months of age as shown in Figure (2).

\section{Discussion}

Sheep and goats usually subjected to the PPRV disease at young age and convalescent period after exposed to infection may be extended for1-2 years [19-21]. Also, Sarker and Islam [22] found that young animals had the highest PPR seroprevalence and they referred that to poor immunity and poor nutrition which predisposed them to PPR. Constable et al. [23] discussed that PPR is most potent in young age (less than one year) due to field infection with some virulent strains of PPR hastened by some bacterial infections such as pneumonic pasteurellosis and entertoxogenic E. coli. 
The disease is considered as one of most economically important disease of livestock $n$ developing countries based on the high morbidity and mortality rates of the viral infection and the small size of the flock [3].

The study results showed that PPR live attenuated vaccine produce strong immunity in vaccinated dams and also in kids and lambs through colostrum. Neutralizing antibodies against PPR in goats and sheep remain up to 3 years also vaccination provides the immunity for up to 3 years. All this information indicates that PPR can be well controlled and eradicated following mass and well planned vaccination programs [24-25].

All vaccinated pregnant dams (5 goats and 5 sheep) in this study showed $100 \%$ of antibody response to the PPR vaccine. Our results also revealed that all vaccinated dams were seroconverted within 2 weeks after vaccination time. These results agree with findings of the study performed in Pakistan and showed that all vaccinated sheep and goats with PPR vaccine were seroconverted [26].

Our study demonstrated that the vaccine is safe. No post vaccinal reaction was observed in the vaccinated animals that means that the virus failed to revert back to virulence. Our results coincide with [27-28].

Our study cleared the loss of maternal immunity in lambs and kids at 120 and 150 days after birth, respectively, which interpreted the registration of high morbidity and mortality in young kids and lambs due to quick decline of maternal antibodies titer in young animals. Our results less or more similar to Abd El-Rahim et al. [29] reported that a relatively high mortality rate among kids and lambs aged 4-9 months.

The study demonstrated that the highest titer averages in kids and lambs were at one month of age then, the titer declined gradually. From 5 lambs, $40 \%$ of lambs were below the protective level at 3 months. At 3.5 months of age, $60 \%$ of lambs became negative and nonprotected; all lambs (100\%) were below the protective level at 4 months of age. At 105 days of age, $20 \%$ of kid became negative. At 120 days after birth, $40 \%$ of kids became unprotected. All kids showed negative nonprotective titer of antibodies at 4.5 months of age. Our result nearly coincide with Bidjeh et al. [30] who demonstrated that the duration of passive immunity in both lambs and kids against PPRV was 4-5 months. Also, Sanne et al. [17] found that a specific titer of antibodies of PPRV had seen in $92 \%$ of totally examined lambs after 15 day of birth. But at 2.5 month of age only $32 \%$ still had antibodies titre against PPRV and at 3 months only 5.3\%had a positive level of antibody.

Therefore, the results obtained from the actual study suggested that the best age for the vaccination of both kids and lambs with PPR live attenuated tissue culture vaccine was at 3 months of age. These findings agree with results obtained by Awa et al., [14] who recommended vaccination at 135 and 105days in kids and lambs, respectively. While, Ata et al. [31] recommended that the vaccination of lambs against PPR was better to do after 90 days of birth. Balamurugan et al. [18] also recommended the kids to be vaccinated against PPR at 120 days of age.

Maternal vaccination is necessary to provide protective passive immunity to the neonate against infections. Live attenuated PPR vaccine is used for sheep and goats and is required to be injected to all animals above the age of 3 month. The vaccine repeated after one year of the primary vaccination before winter season to avoid occurrence of outbreaks especially in endemic regions.

\section{Conclusion}

PPR was found to be more prevalent in young aged small ruminants. Lambs and kids should be vaccinated when the maternal antibodies decline. Vaccination of lambs and kids should be applied at 3 months of age.

\section{Acknowledgements}

The authors thank farmers who participated in this work and technicians for their excellent laboratory assistance and data collections. This work was performed using the facilities of Faculty of Veterinary Medicine, Zagazig University and Dept. of virology, Animal Health Research Institute, Dokki, Giza.

\section{Conflict of interest}

All the authors have no conflict of interest to declare. 


\section{References}

[1] Kumar, N.; Maherchandani, S.; Kashyap, S.K.; Singh, S.V.; Sharma, S.; Chaubay, K.K.; and Ly, H. [2014]: peste des petits ruminants virus infection of small ruminants: a comprehensive review. Viruses. 6 (6):287-327.

[2]Sen, A.; Saravanan, P.; Balamurugan, V.; Bhanuprakash, V.; Venkatesan, G.; Sarkar, J.; Rajak, K.K..; Ahuja, A.; Yadav, V.; Sudhakar, S.B.; Parida, S.; Singh, R.K. [2014]: Detection of subclinical peste des petits ruminants virus infection in experimental cattle. Virus Dis.25 (3): 408-411.

[3]Aitken, I.D. [2007]: Diseases of sheep. 4 ed. Singapore: Blackwell Science: 462466.

[4]Chauhan, H.C.; Lamabade, P.S; Sen, A.; Dadawala, A.L.; Ranaware,P.B.; Chandel, B.; Joshi, D.V.; Patel , S.S.; Pankaj, k.; Shah, N. M. and Kher, H. N. [2011]: The use of pathological and histopathological techniques in the diagnosis of peste des petits ruminants in India. Vet. Ital, 47(1):41-47.

[5]Balamurugan, V.; Hemadri, D.; Gajendragad, M.R.; Singh R.K. and Rahman, H. [2014]: Diagnosis and control of peste des petits ruminants: a comprehensive review. VirusDis. 25(1): 39- 56.

[6]Gibbs, E.P.J.; Taylor, W. P.; Lawman, H.P. and Bryant, J. [1979]: Classification of peste des petits ruminants virus as a fourth member of Genus Morbillivirus. Inter. Virology, 11: 268-278.

[7]De Nardi, M.; LaminSaleh, S. M.; Batten, C.; Oura, C.; Di Nardo, A. and Rossi, D. [2012]: First Evidence of Peste des Petits Ruminants (PPR) Virus Circulation in Algeria (Sahrawi Territories): Outbreak Investigation and Virus Lineage Identification. Transboundary and Emerging Diseases. 59: 214-222

[8]FAO [2009]: Peste des petits ruminants: an increasing threat to small ruminant production in Africa and Asia. EMPRES Transboundary Animal Disease Bullettin No. 33.
[9]Albina, E.; Kwiatek, O.; Minet, C.; Lancelot, R.; De Almeida, R. S and Libeau, G. [2013]: Peste des petits ruminants, the next eradicated animal disease? Veterinary Microbiology, 165: $38-44$.

[10] Zaher, K.S. and Ahmed, W.M. [2014]: Isolation and Identification of Field Isolate of Peste des petits ruminants Virus in Egypt. Global Veterinaria, 12 (5): 667672.

[11]Libeau, G.; Prehaud C.; Lancelot R.; Colas F.; Guerre L.; Bishop, D.; Diallo, A. [1995]: Development of a competitive ELISA for detecting antibodies to the peste des petits ruminants virus using a recombinant nucleoprotein. Research in Veterinary Science, 58: 50-55.

[12]El-Allawy, T. A.; Laisa, S. A.; EL-Rahim, I. H. A.; Abd-EL-Rahim, I. H. A. [1995]: Serological studies on Peste des petits ruminants in Egypt. Proceeding if the second scientific congress Egyptian Society for the cattle Diseases, 5-7 December 1995, Assiut-Egypt, 2: 278289.

[13]Abubakar, M.; Ashiq, S.; Zahoor, A. B.; Arshed, M. J. and Banyard, A. C. [2011]: Diagnosis and control strategies for peste des petits ruminants virus: Global and Pakistan perspectives. Pakistan Veterinary Journal, 31(4): 267-274.

[14]Awa, D. N.; Ngagnou, A.; Tefiang, E.; Yaya, D. and Njoya, A. [2002]: Post vaccination and colostral peste des petits ruminants antibody dynamics in research flocks of Kirdi goats and Eoulbe sheep of north Cameroon. Prevent. Vet. Med 55(4): 265- 271.

[15]Sayem, A. S. M. [2003]: Studies on degradation of antigenicity of PPR vaccine and assessment of its maternal antibody. M. S. Thesis submitted to the Dept. Microbiol. and Hyg. Fac. Vet. Sc. Bangladesh. Agril. Univ.

[16]Ahmed, K.; Jamal, S. M.; Ali, Q. and Hussain, M. [2005]: An outbreak of peste des petits ruminants in goat flock in Okara, Pakistan. Pakistan Veterinary Journal, 25:146-148. 
[17]Sanne, C. B.; Couacy-Hymann, E.; Mathurin, Y. K. and Therese, D. [2006]: Assessment of the duration of maternal antibodies specific to the homologous peste des petits ruminant vaccine "Nigeria $75 / 1 "$ in Djallonkelambs. Nigerian Society for Experimental Biology 18(2):99-103.

[18]Balamurugan, V.; Sen, A.; Venkatesan, G.; Rajak, K.K.; Bhanuprakash, V. and Singh1, R.K. [2012]: Study on passive immunity: time of vaccination in kids Born to goats vaccinated against Peste des petits ruminants. Virologica Sinica, 27 (4):228-233.

[19]Dhar, P.; Sreenivasa, B. P.; Barrett, T.; Corteyn, M.; Singh, R. P. and Bandyopadhyay, S. K [2002]: Recent epidemiology of peste des petits ruminants virus (PPRV). Vet. Microbiol. 88(2):153159.

[20]Ozkul, A.; Akca, Y.; Alkan, F.; Barrett, T.; Karaoglu, T.; Dagalp, S. B.; Anderson, J.; Yesilbag, K.; Cokcaliskan, C.; Gencay, A. and Burgu, I. [2002]: Prevalence, distribution, and host range of Peste des petits ruminants virus, Turkey. Emerg. Infect. Dis. 8(7): 708-712.

[21]Singh, R.P.; Sreenivasa, B.P.; Dhar, P.; Shah, L.C. and Bandyopadhyay, S.K. [2004]: Development of a monoclonal antibody based competitive-ELISA for detection and titration of antibodies to peste des petits ruminants (PPR) virus.Vet.Microbiol.98 (1): 3-15.

[22]Sarker, S. and Islam, H. [2011]: Prevalence and risk factors assessment of PPR in goats in Pajshahi, Bangladesh, Vet. World, 4(12):546- 549.

[23]Constable, P.; Hinchcliff, W. K.; Done, S. and Gruenberg, W. [2017]: Veterinary medicine 11th Edition A textbook of the diseases of cattle, horses, sheep, pigs and goats.

[24]Sen, A.; Saravanan, P.; Balmurugan, V.; Rajak, K.; Sudhakar, S.; Bhanuprakash,
V.; Parida, S. and Singh, R.K. [2010]: Vaccines against PPRV. Expert Review vaccines, (7):785-796.

[25]Saravanan, P.; Sen, A.; Balamurugan, V.; Rajak, K. K.; Bhanupraash, V.; Palaniswami, K. S.; Nachimuthu, K.; Thangarew, A.; Dhinakarraj, G.; Hegde, R. and Singh, K .K. [2010]: comparative efficacy of peste des petits ruminants virus in clinical samples . ActaVirol. (48): 249 255.

[26]Intisar, M.; Ahmad, M.D.; Anjum, A.A and Hanif, A., [2009]: Comparative efficacy of peste des petits rumi-nants (PPR) vaccines available in Pakistan in sheep and goats. Pak. Vet. J., 29: 202-205.

[27]Singh, R. K.; Balamurugan, V.; Bhanuprakash, V.; Sen, A.; Saravanan, p. and Yadav, M.P. [2009]: control and eradication of peste des petits ruminants in sheep and goats in India. Vet Ital .45: 449462.

[28]Singh, R.P.; De, U.K. and Pandey, K.D. [2010]: Virological and antigenic characterization of two peste des petits ruminants vaccine viruses of Indian origin.comp Immunol Microbiol Infect Dis .33 (4): 343 -353.

[29]Abd El-Rahim, I.H.A.; Abdel Baky, M.H.; Habashi, A.R.; Mahmoud, M.M.; Al Mujalii, D.M. [2005]: Peste des petits ruminants among sheep and goats in Saudi Arabia in 2004. Assiut Vet. Med. J. 51 (104), 100-111.

[30]Bidjeh, K.; Diguimbaye, C.; Hendrikx, P.; Dedet, V.; Tehari, D. and Naissingar, S. [1999]: Maternal immunity in young goats or sheep whose dams were vaccinated with anti Peste Des Petits Ruminants vaccine. Cahiers Agric. 8(3): 219-222.

[31]Ata, F. A.; Al-Sumry, H. S.; King, G. J.; Ismail, S. I. and Ata, A. A. [1989]: Duration of maternal immunity to peste des petits ruminants. Vet. Rec.124(22): 590-591. 


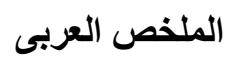

تقيم مده الأجسام المضاده الأموميه الخاصه بمرض طاعون المجترات الصغيره فى الجديان والحملان

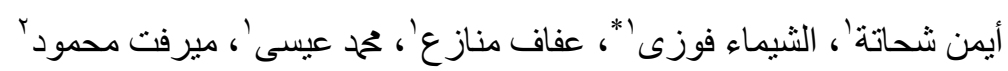

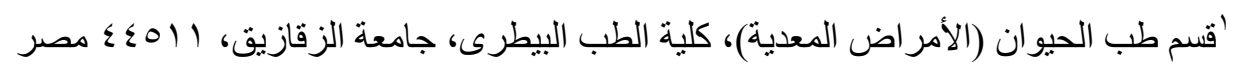

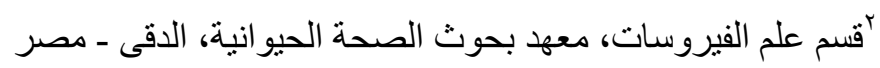

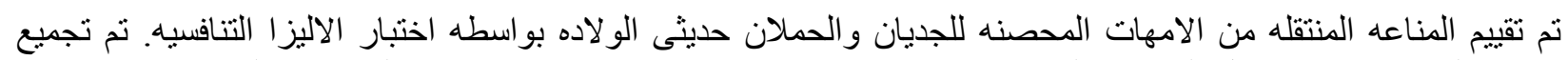

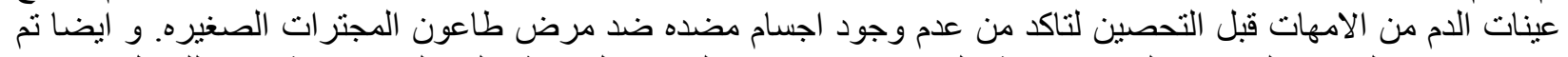

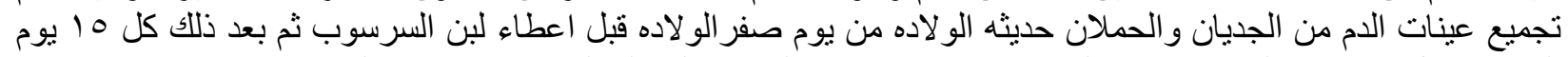

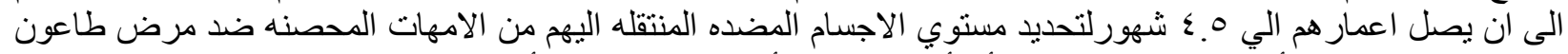

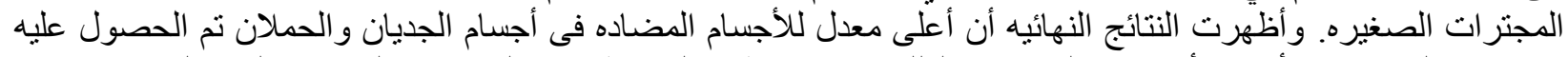

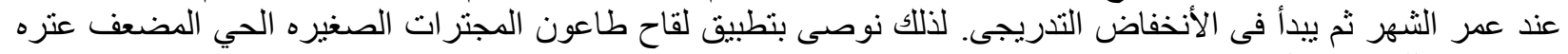

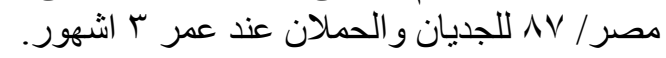

